\title{
Criminologie
}

\section{Débat autour des mesures pour jeunes délinquants}

\section{Jean Lajoie et Marc LeBlanc}

Volume 23, numéro 2, 1990

Après le crime : survivre

URI : https://id.erudit.org/iderudit/017297ar

DOI : https://doi.org/10.7202/017297ar

Aller au sommaire du numéro

Éditeur(s)

Les Presses de l'Université de Montréal

ISSN

0316-0041 (imprimé)

1492-1367 (numérique)

Découvrir la revue

Citer ce document

Lajoie, J. \& LeBlanc, M. (1990). Débat autour des mesures pour jeunes délinquants. Criminologie, 23(2), 107-123. https://doi.org/10.7202/017297ar d'utilisation que vous pouvez consulter en ligne.

https://apropos.erudit.org/fr/usagers/politique-dutilisation/ 
Un chercheur et un praticien discutent des résultats d'une recherche évaluative sur l'efficacité des mesures pour jeunes délinquants.

Jean Lajoie, conseiller au Directeur de la protection de la jeunesse résume d'abord la recherche et apporte ensuite ses commentaires. Enfin, Marc LeBlanc, le chercheur principal, répond aux critiques.

La recherche faisant l'objet de ce débat s'intitule: «L'efficacité des mesures pour jeunes délinquants adoptées à Montréal en 1981: une étude longitudinale». Les auteurs en sont Marc LeBlanc et Hélène Beaumont. Il s'agit d'un texte inédit produit en janvier 1989 par le Groupe de recherche sur l'inadaptation psychosociale chez l'enfant, à l'École de psycho-éducation de l'Université de Montréal.

\section{POINTS SAILLANTS DE LA RECHERCHE (Jean Lajoie)}

Le grand mérite de cette recherche est d'offrir, pour la première fois. un regard critique sur l'efficacité du système de justice des mineurs depuis l'entrée en vigueur de la Loi sur la protection de la jeunesse en 1979 et de la Loi sur les jeunes contrevenants en 1984.

L'intention de départ des auteurs était de se livrer à «la description de l'efficacité relative des mesures pour jeunes délinquants», à partir de l'analyse du processus décisionnel des instances judiciaires et sociales, mais de leur aveu même, «la conclusion majeure qui s'impose d'emblée concerne un sujet différent, la prise de décision».

Ce document interpelle donc les «décideurs» doublement. D'abord au plan de l'efficacité des décisions prises à l'égard des comportements délictueux des adolescents, face à une récidive êventuelle. Ensuite, d'une façon globale, par rapport à la finalité ou à l'objectif fondamental du sentencing, à l'égard de la clientèle adolescente.

Cette recherche se situe dans le sillage d'études antérieures (années 70) notamment sur l'efficacité des programmes d'intervention auprès des jeunes, avant les changements législatifs. Ainsi, on fait un rappel des données globales

* Jean Lajoie, conseiller au Directeur de la protection de la jeunesse, CSSMM, 1001, boul. de Maisonneuve, $5^{\mathrm{e}}$ étage, Montréal, H2L 4 R5.

** Marc LeBlanc, professeur titulaire, École de Psycho-Éducation, Université de Montréal, C.P. 6128, Succ. A., Montréal, H3C 3J7. 
de cette époque à titre d'élément comparatif avec la situation des années 80 .

À l'aide d'un échantillon d'enfants nés entre 1958 et 1962 et qui ont vécu leur adolescence au cours des années 70 , on avait établi que $11 \%$ des garçons de Montréal avaient acquis un dossier criminel avant d'avoir atteint l'âge de 25 ans. De plus, pour ceux qui ont vécu une mesure probatoire ou une mesure d'enfermement au cours de ces années, c'est $70 \%$ qui ont fait l'objet d'au moins une condamnation après avoir atteint l'âge de 18 ans. Plus précisément, c'est $60 \%$ pour la clientèle des probationnaires et $88 \%$ pour celles des centres d'accueil.

La récidive ou la poursuite de la carrière criminelle chez les adolescents à l'âge adulte était donc un phénomène bien documenté avant l'avènement des législations actuelles.

Il s'agit donc d'une recherche longitudinale qui porte sur une clientèle de 577 adolescents déjudiciariés (CSSMM, CSSVM, CSSJ) et de 454 jeunes judiciarisés (Tribunal de la jeunesse, district judiciaire de Montréal) et cela pour des événements signalés entre le 25 mai et le 31 juillet 1981. Il importe de rappeler que nous étions à l'époque du mécanisme de la décision conjointe (CPJ - PDMJ) et que c'était le DPJ qui recevait et statuait sur toutes les demandes d'intenter des procédures.

\section{CARACTÉRISTIQUES DE LA CLIENTÈLE}

Le premier chapitre constitue une étude comparative entre les adolescents déjudiciarisés et judiciarisés. Les caractéristiques des deux clientèles (sociodémographiques et activités délictueuses) sont très différentes. Les jeunes déjudiciarisés «sont plus jeunes et plus souvent à l'école, ils sont plus nombreux à demeurer avec leurs parents et ils ont été moins souvent l'objet d'une mesure antérieure de protection. De plus, l'ampleur de leur activité délictueuse est nettement inférieure à celle des adolescents judiciarisés». En somme, de poursuivre les auteurs, «le DPJ propose la déjudiciarisation pour des adolescents dont l'intégration à leur rôle social est plus évidente et l'activité illicite moins développée» (15\% d'entre eux avaient cependant des antécédents judiciaires).

\section{EFFICACITÉ RELATIVE DES DEUX RÉGIMES}

Le chapitre 2 porte sur l'efficacité relative de la déjudiciarisation et de la judiciarisation. On part de l'hypothèse suivante: les cas déjudiciarisés présentant une meilleure adaptation sociale et une activité délictueuse moindre devraient présenter une meilleure adaptation sociale à l'âge adulte, donc une récidive moindre que les seconds. 
Qu'en est-il? L'étude nous révèle que pour les jeunes judiciarisés, $19 \%$ ont récidivé avant d'atteindre leurs 18 ans et que ce pourcentage passe à $50,1 \%$ à l'âge adulte. Pour les jeunes déjudiciarisés, d'autre part, le taux de récidive se situe à $15,1 \%$ avant l'âge adulte et il grimpe à $44 \%$ après. Et les auteurs de conclure, «la déjudiciarisation n'est pas plus efficace que la judiciarisation pour prévenir la récidive juvénile ou la récidive adulte.»

Afin d'atténuer cette conclusion provisoire et ces performances pour le moins étonnantes, les auteurs ont cherché à savoir si la gravité de la récidive était différente pour chacun des groupes. On découvre, «qu'il s'agisse de la fréquence, de la variété, de la nature des délits et du degré de violence, il n'y a pas de différence statistiquement significative à un niveau suffisant.»

Se fondant sur ces résultats quant à l'abondance et à la nature de la récidive de ces jeunes parvenus à l'âge adulte, les auteurs s'interrogent sur la qualité ou la fiabilité de la sélection des cas fait par le DPJ: «les ARH de la DPJ n'auraient pas sélectionné les adolescents dont les activités délictueuses sont les moins sérieuses et dont la probabilité de récidive est la plus faible.»

Se référant à des études antérieures sur la prédiction de la délinquance, où des facteurs de risque ont abondamment été identifiés et documentés, on met de l'avant l'hypothèse suivante. Si on avait exclu du programme de déjudiciarisation la clientèle possédant des antécédents judiciaires, la récidive adulte passerait de $44 \%$ à $34 \%$. De plus, si on avait exclu les plus âgés ( 16 ans et plus) et les jeunes ne fréquentant pas l'école, le taux de récidive adulte aurait chuté à $29 \%$.

Au terme de ce chapitre, les auteurs en arrivent à porter un jugement sévère sur l'efficacité et même sur la pertinence de maintenir le programme de mesures de rechange, dans sa forme actuelle. «L'effet dissuasif est momentané parce que dans les meilleures conditions, il n'en demeure pas moins que la récidive adulte est le double de la récidive juvénile. Si l'effet dissuasif est momentané compte tenu des types d'adolescents délinquants qui sont déjudiciarisés et si la solution la plus efficace consistait à ne retenir pour la déjudiciarisation que les délinquants occasionnels, la question suivante devient d'actualité: les mesures de rechange sont-elles nécessaires? Si elles responsabilisent les délinquants occasionnels qui affichent une faible probabilité de récidive, leurs coûts financiers sont-ils justifiables? Peut-être qu'il conviendrait d'investir cet argent dans la rééducation et la prévention secondaire des délinquants de transition et/ou de condition?» 


\section{EFFICACITÉ DES MESURES DE RECHANGE}

Le troisième chapitre est consacré à l'efficacité des mesures de rechange, c'est-à-dire à l'influence ou à la performance de l'un ou l'autre type de mesure par rapport à la récidive.

Ainsi, on retrouve pour cette période un taux de fermeture important de $61,5 \%$ tandis que $12,4 \%$ des jeunes ont fait l'objet d'une mesure compensatoire ou réparatrice, $13,5 \%$ d'un suivi social et $9,1 \%$ d'un placement en famille d'accueil ou d'une référence à diverses ressources.

Or, qu'en est-il de l'efficacité de chacune de ces décisions sur la récidive? Pour la fermeture, la récidive juvénile est de $11 \%$ et la récidive adulte fait un bond à $42 \%$. Pour les mesures compensatoires, le score est nettement meilleur: $7 \%$ avant 18 ans et $30 \%$ à l'âge adulte. Le suivi social se solde par une récidive juvénile de $12 \%$ et par une récidive adulte de $49 \%$. Elle est presque identique à la récidive adulte des jeunes ayant fait l'objet d'une probation, soit $50 \%$. Pour les placements en famille d'accueil ou les références, la récidive juvénile est de $39 \%$ et la récidive adulte $46 \%$ (il s'agit ici d'une clientèle très lourde, aux prises avec des problèmes psychosociaux importants).

Les auteurs en arrivent aux conclusions suivantes: l'effet dissuasif des mesures de rechange est de courte durée et ce sont les mesures compensatoires ou réparatrices qui semblent les plus efficaces tant durant l'adolescence qu'à l'âge adulte.

\section{EFFICACITÉ DES MESURES ORDONNÉES}

Parmi les décisions prises par le Tribunal de la jeunesse, on retrouve: l'amende $24 \%$, la probation $19,6 \%$ et le placement en centre d'accueil $13,4 \%$. Les mesures sans intervention (ajournement sine die, sentence suspendue...) comptent pour leur part $24,8 \%$ de l'ensemble des décisions.

La récidive adulte se situe respectivement à $42 \%$ pour les amendes, à $50 \%$ pour la probation, à $69 \%$ pour les placements sous garde et à $55 \%$ pour l'absence d'intervention.

Les auteurs, après avoir analysé largement le processus décisionnel des juges à partir d'un certain nombre de variables, en arrivent à la conclusion suivante: «Ainsi, l'âge et le statut légal de l'adolescent sont des déterminants de l'utilisation de la détention et plus le processus judiciaire s'étendra sur une longue période plus il y aura de chances que les juges ordonnent une mesure restrictive de liberté et il en résultera une récidive plus probable. De fait, si le garçon avait 17 ans et plus lors de la référence au Tribunal et s'il n'était pas sous la responsabilité légale de ses parents, il sera habituellement 
placé en détention pendant la durée du processus judiciaire et dans ces cas la durée du processus tend à dépasser deux mois; pour ces garçons, les juges ordonneront alors plus fréquemment un placement sous garde et la récidive de ces sujets sera quasi certaine.

À l'autre extrême, un adolescent de 16 ans et moins et qui est sous la responsabilité légale de ses parents sera très rarement placé en détention, le processus judiciaire sera court, la décision la plus probable sera l'amende et la récidive de ces sujets sera la plus faible.»

\section{LES CONCLUSIONS}

En guise de conclusion, on retrouve des jugements très sévères sur le nouveau fonctionnement du système de justice des mineurs. «En somme, qu’il s'agisse de l'ancienne législation $L J D$ ou de la nouvelle, $L J C$, les résultats sont tout à fait équivalents. » Et plus loin, «les changements législatifs, administratifs et cliniques majeurs que nous avons connus au Québec depuis la fin des années 1970 n'ont donc pas produit le résultat escompté, réduire la récidive. Il y a autant de récidive maintenant qu'avant et la récidive après 18 ans est relativement équivalente pour la déjudiciarisation et la judiciarisation, pour les diverses mesures de rechange proposées par les ARH de la DPJ et les diverses mesures ordonnées par les juges.»

On fait aussi un certain nombre de suggestions pour améliorer la rigueur du processus décisionnel et cela autant au Tribunal que chez les délégués du DPJ; il y aurait lieu, entre autres, de tenir compte d'une façon systématique de certains facteurs bien identifiés et associés au risque de récidive.

Et on termine sur une lueur d'espoir. «En somme, si nos résultats sur l'efficacité des mesures pour jeunes délinquants portent au désillusionnement, l'analyse des critères de décision laisse de l'espoir puisqu'elle met à jour des constantes qu'il serait facile d'introduire dans la mécanique du système pour mineurs. »

\section{COMMENTAIRES (Jean Lajoie)}

Au terme de la lecture du document, on a appris, en somme, deux choses: l'efficacité des mesures tant judiciaires que sociales est à peu près nulle par rapport à la situation qui prévalait avant les changements législatifs. On apprend, deuxièmement, que ce piètre résultat est imputable au peu de rigueur des processus décisionnels en cause. Le lecteur est enfin invité à s'interroger sur la viabilité de ce new deal et plus particulièrement du Programme de mesures de rechange. 
Cette recherche procède, en fait, à partir du postulat suivant; plus les décideurs seront rigoureux dans le choix de leurs mesures, en se fondant sur des critères largement documentés par la recherche en la matière, plus ils ont de chances d'avoir un impact positif sur la récidive juvénile et adulte.

Les auteurs procèdent également à partir d'un second postulat: l'efficacité d'un système de justice pour mineurs se mesure par sa performance par rapport au taux de récidive qu'elle engendre. En somme, c'est l'objectif ou la finalité du sentencing dont il est question ici.

Nos commentaires seront donc regroupés autour de ces deux postulats.

\section{IMPACT DE LA RIGUEUR DU PROCESSUS DÉCISIONNEL SUR LA RÉCIDIVE}

On ne peut, bien sûr, que souscrire à l'idée que la récidive peut être tributaire pour une bonne part du processus décisionnel; en effet, on peut croire que le fait de prendre la mesure la plus appropriée à la situation en cause aura un impact positif sur son évolution, donc sur la récidive. Mais cet impact n'est que partiel puisque l'administration ou le suivi de la mesure ainsi que l'adhésion de l'adolescent à la mesure sont des facteurs tout aussi déterminants et on n'en rend pas compte dans le texte.

La fiabilité d'un processus décisionnel, d'autre part, repose sur sa rigueur ou sa fiabilité intrinsèque et aussi sur le degré de connaissance qu'en ont les décideurs. Il y a aussi à prendre en compte, du point de vue de l'uniformité de son application, le nombre de personnes qui en sont responsables; en effet, plus il y a de personnes à prendre des décisions, plus le risque de disparité est grand puisqu'un tel processus implique un certain degré de subjectivité et une certaine marge de discrétion.

Et il y a, ici, des précisions importantes à apporter au contexte qui prévalait, en 1981, au moment où la collecte des données a été faite, et qui ont un impact majeur sur la fiabilité des résultats obtenus ainsi que sur le bienfondé de certains jugements critiques sur les pratiques du DPJ.

Il faut donc savoir qu'au CSSMM on a assisté, à partir de janvier 1979, à une prolifération quasi démentielle d'intervenants affectés à l'évaluation des situations prévues à l'article $\mathbf{4 0}$ de la Loi sur la protection de la jeunesse et que cette situation a prévalu jusqu'en avril 1984 .

Cette fonction dévolue jusque-là aux agents de probation s'est retrouvée disséminée à travers tous les points de service du CSSMM, le Service de probation ayant été aboli au cours de cette période. C'est ainsi que tous les intervenants impliqués auprès de la clientèle adolescente, dans un contexte de pro- 
tection (article 38) ou dans le cadre de la LSSSS, étaient aussi appelés à prendre des décisions, en vertu de l'article 40 , lorsque le jeune faisait l'objet d'un signalement en vertu de cet article. Ces intervenants, qui n'avaient à peu près pas de connaissances de la dynamique du délinquant ni du système judiciaire, en étaient souvent réduits à prendre des décisions uniquement en fonction des problématiques qui n'avaient rien à voir avec la délinquance. De plus, ils n'avaient reçu aucune information sur la grille d'orientation qui venait d'être mise au point et à laquelle on fait référence dans la recherche. Il y avait toutefois les anciens agents de probation, disséminés dans les équipes enfance-famille, qui possédaient cette expertise en délinquance et qui avaient en main cette grille d'orientation.

Il faut cependant avouer qu'une bonne concentration des «articles 40 » (les nouveaux cas, soit les jeunes, ne faisant l'objet d'aucune prise en charge) se retrouvaient au Service Jeunes contrevenants. Mais encore là, on se retrouvait face à une situation paradoxale du point de vue de l'uniformité de la prise de décision. On y retrouvait trois équipes ayant des mandats fort différents. L'équipe dite de Réception et traitement des signalements prenaient les décisions pour les infractions aujourd'hui sanctionnées par la Loi sur les poursuites sommaires (đébit de boisson, code de la route,....) et pour des délits mineurs (vols à l'étalage,...).

L'équipe dite Intervention minimale avait la responsabilité de l'application des mesures volontaires (aujourd'hui mesures de rechange) et on lui acheminait les jeunes dont les délits étaient de gravité moyenne et susceptibles d'entreprendre une mesure réparatrice ou compensatoire.

L'équipe dite Évaluation-orientation écopait des délits graves et susceptibles d'être référés au Tribunal de la jeunesse.

À l'intérieur d'un tel réseau décisionnel, les risques de distorsion étaient énormes. Les délits faisaient l'objet d'une présélection sur dossier — donc grossière - et leur classification selon l'ordre de gravité était fort relative. Donc, pour un même type de délit, on pouvait avoir une propension à la déjudiciarisation ou à la judiciarisation selon que l'on ouvrait à l'intérieur de l'une ou l'autre équipe ou de l'un ou l'autre registre de gravité.

Cette dispersion du processus décisionnel a cependant été corrigée en avril 1984 avec l'implantation de la LJC. C'est l'équipe Intervention minimale qui est responsable à elle seule de plus de $90 \%$ des orientations dans le cadre du Programme de mesures de rechange. La partie infime qui lui échappe est assumée par les délégués à la probation dont le nombre a été restreint à une quinzaine. 
Il importe de faire une dernière observation sur le contexte décisionnel qui prévalait en 1981 et elle concerne la philosophie d'intervention.

On sait que les «mesures volontaires» avaient été introduites dans la loi 24 dans la foulée des divers projets de déjudiciarisation dont les objectifs étaient forts variables de l'un à l'autre. De plus, la délinquance se voyait octroyer un traitement qui se voulait différent du modèle médical de la Loi sur les jeunes délinquants et qui se voulait également plus soucieux du respect des droits des jeunes. Mais la philosophie demeurait sensiblement la même; la protection de l'enfant conservait toute sa préséance sur la protection de la société. Le principe «de la compromission de la sécurité ou du développement» de l'enfant demeurait omniprésent.

Ce n'est qu'au fil des années et avec l'avènement de la Loi sur les jeunes contrevenants, que la notion de responsabilisation a émergé véritablement. La loi 24 a donc perpétué, de 1979 à 1984, une ambiguité qui a fini par s'estomper avec la Déclaration de principes de l'article 3 de la LJC.

La grille d'orientation, à laquelle on fait souvent référence et qui avait été conçue à la fin de l'année 1979, était également tributaire de cette philosophie et de cette ambiguité. Il a fallu quelques années avant qu'une nouvelle grille, davantage en accord avec l'esprit de la LJC, ne fasse surface; elle vit le jour, en fait, en 1986. Les indications demeurent sensiblement les mêmes, mais leur articulation par rapport à la prise de décision est beaucoup plus rigoureuse ${ }^{1}$.

Donc, si on considère la situation qui prévalait en 1981, au moment de la collecte des données, le nombre considérable des décideurs, la diversité de leur champ de pratique, leur manque de connaissances en délinquance, l'ambiguité des objectifs de la loi 24, l'état expérimental de la grille d'orientation et sa quasi-absence de diffusion, il n'est pas étonnant que l'on en soit arrivé aux distorsions et aux piètres résultats dénoncés par les auteurs.

Mais ce qui est inacceptable, c'est qu'on mette en doute la viabilité et l'avenir de l'actuel Programme de mesures de rechange en faisant une extrapolation à partir d'une situation qui prévalait, il y a huit ans, et qui n'est absolument plus la même.

1. Une nouvelle grille vient d'être mise au point, en mars 1990 , et elle est au stade expérimental. 


\section{LES OBJECTIFS OU LA FINALITÉ DE LA PRISE DE DÉCISION}

Mesurer l'efficacité du système de justice pour mineurs par sa performance sur la récidive, c'est d'abord la réduire à une seule dimension et c'est aussi en questionner les objectifs ou la finalité.

L'objectif du sentencing a donné lieu à une littérature abondante en droit pénal. On en dénombre en général cinq: la dissuasion, la réadaptation, la neutralisation, le rétributisme et la dénonciation. Chacun d'eux a un sens très spécifique : éviter qu'un tel comportement ne se reproduise, permettre au délinquant de se réinsérer dans la société, protéger la société, en incarcérant (habituellement) le contrevenant, imposer une conséquence à l'acte d'une façon proportionnelle au préjudice subi et faire état de la réprobation sociale par rapport à un type de crime en particulier par l'imposition d'une sentence exemplaire.

La plupart du temps, une même sentence vise plus d'un objectif: elle devrait, en principe, tenir compte de l'ensemble des éléments du comportement délictueux et des besoins de chaque individu. Ainsi le cadre décisionnel, auquel font référence les auteurs de la recherche, devient quelque chose de très complexe puisqu'il procède à partir de différents objectifs possibles et qu'il est tributaire, pour une large part, de l'objectif poursuivi.

Dans le cadre de la Loi sur les jeunes contrevenants, on retrouve d'une façon très explicite deux grands objectifs dans la Déclaration de principes: la protection de la société (la neutralisation) et la réponse aux besoins de l'adolescent, compte tenu de son degré de maturité (la réadaptation). Selon que l'on poursuive l'un ou l'autre objectif, la décision du Tribunal ou du DPJ le concernant pourrait être fort différente, et les décisions possibles à son égard pourraient même être incompatibles entre elles selon que l'on privilégie l'un ou l'autre.

Le Rapport de la Commission canadienne sur la détermination de la peine, que citent fréquemment les auteurs, pose bien le problème de la relativité du processus décisionnel: «Il y a cependant parfois une incompatibilité réelle entre les objectifs pénaux traditionnels. Ainsi la jeunesse d'un contrevenant (son degré de maturité) est une circonstance atténuante dans une logique de la réadaptation: elle devient cependant une circonstance aggravante dans le cadre d'une logique de la neutralisation sélective, puisqu'elle est considérée comme l'un des facteurs les plus fiables pour prévoir les risques futurs de récidive." (page 146).

En somme, dans une perspective de réadaptation, plus un adolescent est jeune plus on devrait tenir compte de ses besoins, car les possibilités de 
changements sont plus grands, et cela en acceptant de courir le risque qu'il récidive, donc de mettre en cause la protection de la société.

À l'opposé, si on suit une logique de neutralisation, plus il sera jeune, plus il faudra être sévère, en privilégiant, par exemple, une politique d'enfermement car le risque de récidive est plus grand.

Neutralisation vs réadaptation, résume bien le débat que se livrent, «sur le terrain", les tenants de l'une et l'autre tendance. C'est donc dire que s'entendre sur un cadre décisionnel n'est pas une mince affaire, car il sera toujours tributaire des philosophies en cause.

On peut donc dire, pour conclure ce commentaire, que réduire l'objectif du cadre décisionnel et l'efficacité du système de justice pour mineurs par leur seul impact sur la récidive est un choix un peu facile et que cela, de plus, ne tient pas du tout compte de la complexité et de l'imprévisibilité (capacité de changement) liées à la réalité de l'adolescence.

Ce parti pris est enfin incompatible avec la philosophie dont le législateur a voulu doter la Loi sur les jeunes contrevenants.

\section{CONCLUSIONS}

Malgré ces commentaires sévères sur les conclusions un peu hâtives des auteurs de la recherche, quant à la performance du système de justice des mineurs au Québec, ce document demeure extrêmement intéressant à plus d'un point de vue.

C'est d'abord la première recherche évaluative du genre. Il nous offre aussi des données abondantes sur la récidive qui, même si elle n'est pas le seul critère d'évaluation de l'efficacité de notre système, demeure un élément majeur. On prend conscience enfin de l'importance de développer un cadre décisionnel rigoureux à partir d'indicateurs bien documentés par la recherche empirique.

Ce document mérite donc une lecture attentive et devrait faire l'objet d'une réflexion collective de la part des directeurs provinciaux et des délégués à la jeunesse. 


\section{RÉACTIONS AUX COMMENTAIRES: L'INSTANTANÉITÉ ET L'INUTILITÉ DES RÉSULTATS DE LA RECHERCHE ÉVALUATIVE (Marc LeBlanc)}

Jean Lajoie résume les points saillants de notre rapport de recherche sur l'efficacité des mesures pour jeunes délinquants et il en commente les conclusions. Son résumé est exact et fidèle. Ses commentaires soulèvent des questions importantes. Toutefois, il succombe subrepticement au réflexe technocratique habituel qu'induisent les résultats des recherches évaluatives. En fait, il minimise leur pertinence en soutenant que la situation a changé, et il réduit leur utilité en arguant que le critère de la récidive est limité. Je le remercie de le faire par écrit, car cela me donne l'occasion d'élaborer des conclusions qui étaient peut-être trop sèches dans notre rapport de recherche.

\section{LA SITUATION A CHANGÉ}

Lorsque je présente les résultats de mes recherches évaluatives à des gestionnaires et à des praticiens, le même argument revient sous une forme ou une autre: «ce n'est plus comme cela maintenant». On m'a servi l'argument au terme de l'évaluation de Boscoville (LeBlanc, 1983), des ateliers de travail (LeBlanc et Trudeau-Le Blanc, 1988, 1989), des mesures de placement au cours des années 1970 (Le Blanc, 1985, 1986) et de la désinstitutionnalisation (Le Blanc et Beaumont, 1987). Jean Lajoie utilise le même argument mais il est plus rigoureux que la majorité de ses confrères. Il renvoie à six preuves pour minimiser la pertinence de nos résultats. Reprenons chacune d'entre elles de manière à vérifier si la situation en 1981 était tellement désastreuse que nos résultats, quoique intéressants sur le plan historique, se sont démodés sur le plan pratique.

Le nombre considérable de décideurs. En 1981, il n'y avait qu'un service de réception des signalements acheminés par les policiers pour l'article 40 , et les signalements retenus étaient traités par 24 agents de relations humaines (ARH) (Le Blanc et Beaumont, 1985). Accordons, par ailleurs, que les nouveaux signalements, pour des adolescents de 14 à 18 ans déjà pris en charge, étaient traités par le personnel de d'autres organismes, et représentaient un nombre infime des cas étudiés par notre recherche. Ce nombre d'une vingtaine de décideurs n'est sûrement pas si considérable qu'il soit impossible pour eux d'atteindre un certain degré de consensus et d'uniformité. À ma connaissance, ce nombre de décideurs n'est pas sensiblement différent à l'heure actuelle. Voilà un premier argument qui n'a pas beaucoup de poids.

La diversité de leurs champs de pratique. En 1982, à l'occasion d'une enquête auprès des ARH de la PDJ, nous ne constations pas une très grande 
diversité des champs de pratique (Le Blanc, 1984). $87 \%$ des ARH possédaient un diplôme universitaire, dont $76 \%$ obtenus en service social ou en criminologie; ils avaient accumulé en moyenne sept années d'expérience avec des jeunes délinquants et la grande majorité des ARH déclaraient utiliser comme critères de décision, dans l'ordre, les relations familiales, la délinquance, les amis et les problèmes personnels de l'adolescent. Comment ne pouvaient-ils pas s'entendre sur une grille de décision uniforme avec toute cette formation et cette expérience commune? La diversité des champs de pratique n'a sûrement pas beaucoup changé depuis 1981 puisque la mobilité du personnel n'est pas impressionnante au CSSMM.

Un manque de connaissances sur la délinquance. Cet argument est surprenant compte tenu de la formation de base des ARH décrite ci-dessus, et du fait qu'ils étaient conseillés et dirigés pour la plupart par des criminologues. Cela laisse supposer que la formation de base était inadéquate ou que les connaissances acquises n'étaient pas mises en application. En 1981, les principales études sur la prise de décision avaient eu lieu comme en fait foi la liste des références de notre rapport, et des sessions de formation avaient été tenues avec la plupart des ARH du Québec sur les résultats des recherches pertinentes provenant du Québec ou d'ailleurs. Il faut donc chercher ailleurs les raisons de l'absence de spécificité et d'uniformité dans les décisions.

L'état expérimental de la grille. La grille de la Table de consultation et de concertation publiée en 1980 était non seulement expérimentale, comme la qualifie Jean Lajoie, mais elle avait le défaut d'être un inventaire relativement exhaustif des informations pertinentes pour évaluer un jeune délinquant sans indiquer les critères primordiaux d'un pronostic de délinquance répétitive. Malgré que la grille actuelle élague parmi les indicateurs (Service Jeunes contrevenants, 1989), elle n'est pas plus explicite; elle ne précise pas mieux les indicateurs de l'activité délictueuse (fréquence, durée, aggravation, etc.), des facteurs familiaux (supervision, communication, etc.), scolaires, etc.

La quasi-absence de diffusion d'informations sur la JPF. Cette observation ne concorde pas avec l'évaluation de la Commission Charbonneau (1982) qui parle de «préparatifs intenses», sans compter les colloques et les sessions d'information diverses tenues en 1980 et 1981. La documentation que $\mathrm{j}$ 'ai lue et les témoignages que $\mathrm{j}$ 'ai entendus concernant cette question au moment de la Commission Charbonneau, ainsi que la lecture du rapport de la Mission d'implantation de la Loi sur les jeunes contrevenants et de la Loi sur la protection de la jeunesse (1985) me portent à croire que l'ampleur des activités n'a pas été tellement différente pour l'une ou l'autre loi. 
L'ambiguïté de la Loi 24 . Jean Lajoie aurait dû dire «la confusion légale» introduite par la Loi 24, qui mettait de côté la vérification de la suffisance des preuves, le contrôle des obstacles de droit et la qualification du délit. Voici un changement important qui a été introduit: c'est le procureur de la couronne qui reçoit dorénavant les demandes d'intenter des procédures de la part des policiers, alors qu'en 1981 c'était les ARH de la DPJ. Toutefois, l'étude de Trépanier et Gagnon (1987) indique que le tamisage par les procureurs était traditionnellement minimal. Compte tenu de cette observation, il est peu probable que le changement dans le mécanisme d'orientation introduit par la LJC ait des conséquences qui dépassent la question de la légalité; en effet, dans notre rapport, nous citons des chiffres officiels du ministère de la Justice du Québec (1987) qui indiquent qu'avant et après le changement législatif la proportion des adolescents judiciarisés est sensiblement la même; de plus, l'analyse des données statistiques que rapporte Thibault (1989) confirme. toute proportion gardée, les tendances antérieures si l'on fait exception de l'année 1988, une année exceptionnelle en comparaison des précédentes en ce qui concerne son taux de judiciarisation. Un changement de processus qui ne produit pas un effet notable pendant plusieurs années sur les résultats n'est sûrement pas un changement réel.

En somme, les arguments de Jean Lajoie pour lancer l'épouvantail «ce n'est plus comme cela maintenant» ne résistent pas longtemps à des faits qu'il oublie de prendre en compte. Il a donc peu de munitions pour appuyer son argument à l'effet que nous ne pouvons «mettre en doute la viabilité et l'avenir de l'actuel programme de mesures de rechange en faisant une extrapolation à partir d'une situation qui prévalait, il y a huit ans, et qui n'est absolument plus la même». En fait, l'extrapolation est sûrement limitée parce que les changements ne sont probablement pas aussi considérables que le technocrate nous le laisse entendre.

Ce que nous avons voulu faire dans nos conclusions, c'est distinguer entre la viabilité, l'utilité et la nécessité des mesures de rechange. Nous croyons avec Jean Lajoie qu'elles sont viables, et qu'elles peuvent durer et se développer, comme c'est le cas depuis dix ans. Elles sont peut-être utiles, et satisfont sûrement un besoin d'éducation pour les adolescents et de rétribution pour la société; à ma connaissance il s'agit d'un postulat non prouvé et difficilement démontrable.

Par contre, nous mettons en doute la nécessité d'un tel programme pour une seule raison: si les mesures de rechange sont appliquées aux adolescents les moins délinquants, leur pronostic, en fait leur probabilité de récidive est telle qu'il s'agit d'un investissement peu rentable socialement; les ressources limitées dont dispose le réseau des Affaires sociales devraient être concentrées 
sur les délinquants entre 15 et 30 ans qui récidivent à répétition, engorgent le système de justice et qui, surtout, sont si difficiles à réadapter. Si l'ampleur et la qualité des ressources sont suffisantes pour ces délinquants, ce que les spécialistes de la réadaptation mettent en doute, alors les mesures de rechange pourraient être développées. Nous adoptions donc une position qui mettait l'accent sur la rentabilité des mesures de rechange dans une société de rareté.

Si les mesures de rechange apparaissent viables sur le plan organisationnel, d'une utilité incertaine pour la société et superflues pour les jeunes contrevenants, il ne faut pas oublier qu'elles remplissent une fonction essentielle pour les intervenants. Elles maintiennent leur moral parce que les jeunes qui en font l'objet sont moins rébarbatifs, les parents plus collaborateurs et les succès sont aussi plus immédiats et évidents.

\section{LA RÉCIDIVE EST UN CRITĖRE INAPPROPRIÉ}

Jean Lajoie nous reproche le recours au critère de la récidive qu'il trouve «un peu facile» et «qui ne tient pas compte de la complexité ... de la réalité de l'adolescence». Ces critiques sont conceptuellement justes mais pratiquement injustifiées.

La récidive, un critère facile. Depuis longtemps les criminologues considèrent que la récidive constitue un critère insuffisant et ils utilisent d'autres critères d'adaptation sociale ainsi que divers critères d'adaptation personnelle. Nous l'avons aussi fait dans nos diverses recherches évaluatives (voir les références). Malheureusement, quel que soit le critère (l'appréciation subjective de l'adolescent, des questionnaires de personnalité, des indices de l'adaptation sociale, la nature de la récidive, etc.), les résultats obtenus sont équivalents : les individus les plus délinquants conservent leur position de sujets les plus inadaptés quel que soit le critère. Donc, la récidive n'est pas un critère facile, mais il s'agit d'un critère qui représente adéquatement la complexité de la réalité de l'adaptation des délinquants.

La récidive, un critère représentatif. Jean Lajoie utilise les fonctions de la peine (dénonciation, dissuasion, rétribution, neutralisation et réadaptation) et les objectifs énumérés au préambule de la Loi sur les jeunes contrevenants pour soutenir que la récidive ne permet pas de mesurer l'atteinte de toutes ces finalités. D'une part, il limite la finalité de la protection de la société à la fonction neutralisation de la peine, alors qu'elle peut aussi bien être atteinte par la dénonciation, la dissuasion et la rétribution. D'autre part, il circonscrit la finalité de répondre aux besoins des adolescents à la fonction de réadaptation, alors que la dissuasion peut aussi être une réponse adéquate aux besoins du délinquant. Les écrits de Cusson $(1983,1987)$ sont fort éclairants sur ces sujets. De plus, il oublie de rappeler que les fonctions de prononcé de la peine, 
la dénonciation, et de l'exécution de la peine, la neutralisation et la rétribution sont toujours poursuivies conjointement avec les fonctions relatives aux conséquences de la peine, la dissuasion et la réadaptation. Une ordonnance de placement en milieu sécuritaire dénonce le crime, neutralise le délinquant, rétribue la société, dissuade le délinquant de recommencer, et le réadapte peut-être.

Il est juste de penser que la récidive ne permet qu'indirectement de mesurer la manière dont les fonctions de neutralisation et de rétribution sont remplies; par contre, la récidive mesure directement les résultats des fonctions relatives aux conséquences de la peine, la dissuasion et la réadaptation du délinquant, qui sont les plus fondamentales parce qu'elles relèvent à la fois des finalités de la protection de la société et de la réadaptation des délinquants. La récidive n'est donc pas le choix le plus facile, mais c'est le choix le plus logique; et, ce critère, plutôt que d'opposer les deux finalités de la Loi sur les jeunes contrevenants, les réconcilient.

Si la récidive est un critère éminemment pratique des finalités de la peine, une autre raison milite en faveur de son utilisation. Considérant que moins de $20 \%$ des criminels produisent plus de $50 \%$ des délits, au Québec comme ailleurs dans le monde (Le Blanc et Fréchette, 1989), ce sont donc les récidivistes qui constituent le groupe cible à considérer si l'on veut s'attaquer au couur du problème de la délinquance. La récidive devient ainsi un critère approprié pour l'évaluation de l'efficacité des mesures pour jeunes délinquants.

\section{CONCLUSION}

Les conclusions que nous avons énoncées dans notre rapport ne nous apparaissent donc ni sévères ni hâtives comme Jean Lajoie le prétend. Elles étaient plus réalistes qu'il ne le laisse entendre. En effet, nos contre-arguments montrent que les changements n'étaient pas si fondamentaux au niveau des prises de décisions, et que la récidive demeure le critère qui s'applique à plusieurs des fonctions de la peine. Ce que nous soutenons en fait dans notre rapport, c'est que les modifications législatives et organisationnelles que nous avons expérimentées n'ont pas nécessairement été accompagnées de transformations dans les pratiques des intervenants.

Pourquoi les gestionnaires et les praticiens adoptent-ils une attitude défensive lorsque les résuitats des recherches évaluatives leur sont transmis? Plutôt que de balayer les résultats du revers de la main et de se retrancher dans un corporatisme technocratique, pourquoi n'optent-ils pas pour une attitude de développement? Une attitude qui consiste à se demander quel sont les résultats 
qui pourraient justifier des modifications dans les pratiques, ou tout au moins des expérimentations de manière à vérifier la pertinence des recommandations qui en résultent. Il ne s'agit pas d'accepter d'emblée les recommandations qui découlent des recherches, il s'agit plutôt de les considérer avant de les oublier.

Peut-être qu'une telle attitude permettrait d'éviter certaines erreurs et de progresser plus rapidement. Peut-être qu'une attitude de recherche de critères permettant la systématisation des décisions rendrait mieux justice aux adolescents, et réduirait les délais dans la prise de décisions. Ce sont les objectifs que nous poursuivons tous et c'est la collaboration qu'offrent, peut-être malhabilement, les chercheurs. Une philosophie d'aide aux jeunes et de justice nous apparait tout à fait compatible avec la recherche de la rigueur dans les prises de décisions.

\section{RÉFÉRENCES}

CHARBONNEAU, J. P. (1982), Rapport de la Commission parlementaire spéciale sur la protection de la jeunesse. Québec, Assemblée nationale, Commission parlementaire spéciale sur la protection de la jeunesse.

CUSSON, M. (1983), Le contrôle social du crime, Paris, Presses universitaires de France.

CUSSON, M. (1987), Pourquoi punir? Paris, Dalloz.

LE BLANC, M. (1983), Boscoville, la rééducation évaluée, Montréal, HMH.

LE BLANC, M. (1984), «Les agents de relations humaines des CSS, la Loi sur les jeunes contrevenants et le système de justice pour mineurs». Service Social, 33, 2-3: 324-356.

LE BLANC, M. (1985), "De l'efficacité d'internats québécois», Revue canadienne de psychoéducation, 14, 2: 113-120.

LE BLANC, M., (1986), "Réussite sociale et inadaptation: épidémiologie prospective», Revue canadienne de criminologie, $28,4: 363-377$.

LE BLANC, M., BEAUMONT, H. (1985), Description du fonctionnemen du rribunal de la jeunesse de Montréal entre mai 1981 et avril 1982. Rapport final et annexes techniques. Montréal, Cérij.

LE BLANC, M., BEAUMONT, H., (1987), La réadaptation dans la communauté au Québec : inventaire des programmes. Rapport soumis à la Commission d'enquête sur les services de santé et les services sociaux. Montréal, Centre international de criminologie comparée, Université de Montréal.

LEBLANC, M., BEAUMONT, H. (1989), L'efficacité des mesures pour jeunes délinquants adoptées à Montréal en 1981, une étude longitudinale. Montréal, Groupe de recherche sur l'inadaptation psycho-sociale à l'enfance, Université de Montréal.

LE BLANC, M., FRÉCHETTE, M. (1989), Male Criminal Activity, From Childhood Through Youth: Multilevel and Developmental Perspectives, New York, Springer-Verlag. 
LE BLANC, M., TRUDEAU-LE BLANC, P. (1988), «Le travail, une alternative à la scolarisation pour jeunes mésadaptés socio-effectifs I: les formules d'atelier et leurs mises en œuvre», Revue canadienne de psycho-éducation, 17, 2: 105-119.

LE BLANC, M., TRUDEAU-LE BLANC, P. (1989), «Le travail, une alternative à la scolarisation pour jeunes mésadaptés socio-effectifs II : les impacts et une politique d'action", Revue canadienne de psycho-éducation, 18, 1: 1-20.

MINISTÈRE DE LA JUSTICE DU QUÉBEC (1987), Application du programme de mesures de rechange. Gouvernement du Québec, ministère de la Justice, Direction des Affaires criminelles et pénales, Direction du secteur jeunesse.

Mission québécoise d'implantation de la Loi sur les jeunes contrevenants et de la Loi sur la protection de la jeunesse (1985), Rapport des activités de la mission québécoise d' implantation de la Loi sur les jeunes contrevenants et des amendements apportés à la Loi sur la protection de la jeunesse, Québec. Table centrale de consultation et de concertation. Loi sur la protection de la jeunesse.

Service Jeunes contrevenants (1989), L'évaluation-orientation dans le cadre du programme des mesures de rechange. Loi sur les jeunes contrevenants, Équipe intervention minimale, Service Jeunes contrevenants. Direction de la protection de la jeunesse. Centre des services sociaux du Montréal métropolitain.

Table centrale de consultation et de concertation. Loi sur la protection de la jeunesse (1980), Évaluation-orientation, les jeunes contrevenants, Québec, Table centrale de consultation et de concertation, Loi sur la protection de la jeunesse.

THIBAULT, L. (1989). Loi sur les jeunes contrevenants: délinquance juvénile et interventions auprès des jeunes contrevenants au CSSMM. Montréal. Centre des services sociaux du Montréal métropolitain.

TRÉPANIER, J., GAGNON, R. (1987), La déjudiciarisation à la Cour du bien-être social entre 1971 et 1976. Rerue canadienne de psycho-éducation. 16. 2: 77-90. 